\title{
Secretion of a foreign protein from budding yeasts is enhanced by cotranslational translocation and by suppression of vacuolar targeting
}

\author{
Ivy Fitzgerald ${ }^{1}$ and Benjamin S Glick ${ }^{2^{*}}$
}

\begin{abstract}
Background: Budding yeasts are often used to secrete foreign proteins, but the efficiency is variable. To identify roadblocks in the yeast secretory pathway, we used a monomeric superfolder GFP (msGFP) as a visual tracer in Saccharomyces cerevisiae and Pichia pastoris.

Results: One roadblock for msGFP secretion is translocation into the ER. Foreign proteins are typically fused to the bipartite a-factor secretion signal, which consists of the signal sequence followed by the pro region. The a-factor signal sequence directs posttranslational translocation. For msGFP, posttranslational translocation is inefficient with the a-factor signal sequence alone but is stimulated by the pro region. This requirement for the pro region can be bypassed by using the Ost1 signal sequence, which has been shown to direct cotranslational translocation. A hybrid secretion signal consisting of the Ost1 signal sequence followed by the a-factor pro region drives efficient translocation followed by rapid ER export. A second roadblock for msGFP secretion in S. cerevisiae occurs during exit from the Golgi, when some of the msGFP molecules are diverted to the vacuole. Deletion of the sorting receptor Vps10 prevents vacuolar targeting of msGFP at the expense of missorting vacuolar hydrolases such as carboxypeptidase Y (CPY) to the culture medium. However, a truncation of Vps10 blocks vacuolar targeting of msGFP while permitting CPY to be sorted normally.

Conclusions: With budding yeasts, if the secretion or processing of a foreign protein is poor, we recommend two options. First, use the Ost1 signal sequence to achieve efficient entry into the secretory pathway while avoiding the processing issues associated with the a-factor pro region. Second, truncate Vps10 to suppress diversion to the vacuole. These insights obtained with msGFP highlight the value of applying cell biological methods to study yeast secretion.
\end{abstract}

Keywords: Yeasts, Secretion, Heterologous protein production, Signal sequence, Alpha-factor, Translocation, Sorting, Vacuole, Ost1, Vps10

\section{Background}

Budding yeasts such as Saccharomyces cerevisiae and Pichia pastoris are widely used as hosts to produce foreign proteins for research and therapeutic purposes [1-5]. Many of these foreign proteins traverse the secretory pathway $[6,7]$. Entry into the secretory pathway requires an $\mathrm{N}$-terminal signal sequence that directs translocation into the endoplasmic reticulum (ER). A typical signal sequence includes a stretch of hydrophobic residues, followed by a cleavage site that is recognized by signal

\footnotetext{
*Correspondence: bsglick@uchicago.edu

${ }^{2}$ Department of Molecular Genetics and Cell Biology, University of Chicago,

920 East 58th Street, Chicago, Illinois 60637, USA

Full list of author information is available at the end of the article
}

peptidase in the ER lumen. After entry into the ER, a protein folds, and may undergo additional changes that include disulfide bond formation, glycosylation, and oligomerization. Finally, the protein is delivered from the ER to the Golgi apparatus and then to the extracellular space. For different foreign proteins expressed in yeasts, the level of secreted product varies widely, presumably because specific steps in the secretory pathway can be inefficient [3]. Much interest is focused on identifying and overcoming these roadblocks.

One source of variability is the secretion signal. A number of signal sequences have been used to secrete foreign proteins from yeasts, but the most popular is the secretion signal from $S$. cerevisiae pre-pro- $\alpha$-factor, which 
is the precursor to a peptide mating pheromone $[1,8,9]$. Pre-pro- $\alpha$-factor contains a 19-residue signal sequence that terminates in a signal peptidase cleavage site. Following the signal sequence is a 66-residue pro region, which is removed in the late Golgi by the Kex2 endoprotease [10]. Finally, downstream of the dibasic Kex2 cleavage signal is an EAEA tetrapeptide, which is trimmed by the dipeptidyl aminopeptidase Ste13 [11]. Efficient secretion of foreign proteins requires the entire pre-pro- $\alpha$-factor secretion signal, with or without the downstream EAEA tetrapeptide, but the secreted products are often heterogeneous due to incomplete processing by Kex2 or Ste13 [1,5].

The reason for the effectiveness of the complete prepro- $\alpha$-factor secretion signal has been unclear. Standard assays for protein secretion simply measure the amount of product that reaches the culture medium. To examine earlier steps in the secretory pathway, we employed a monomeric superfolder variant of green fluorescent protein (msGFP) as a model foreign protein. Visualization of intracellular msGFP indicated that with the $\alpha$-factor signal sequence, which mediates posttranslational translocation across the ER membrane $[12,13]$, the translocation step is inefficient. More efficient posttranslational translocation was obtained by inclusion of the $\alpha$-factor pro region. Based on this insight, we used the Ost1 signal sequence to drive cotranslational translocation of msGFP [14], thereby bypassing the pro region and its associated complications.

After entering and exiting the ER, a protein can nevertheless fail to be secreted if it is diverted to the vacuole. Budding yeasts contain a vacuolar sorting receptor called Vps10 that recognizes the carboxypeptidase Y (CPY) precursor as well as certain misfolded proteins [15-20]. When msGFP was targeted to the secretory pathway in S. cerevisiae, some of the msGFP molecules reached the vacuole in a Vps10-dependent manner, confirming a report that the quality control function of Vps10 extends to folded GFP [21]. Building on a previous functional dissection of Vps10 [15], we found that deletion of a single domain of Vps10 prevented diversion of msGFP to the vacuole without causing missorting of CPY. This approach may enable the engineering of yeast strains that efficiently secrete foreign proteins while preserving normal vacuolar function.

\section{Results}

The pro region enhances secretion even in the absence of Erv29

GFP folds poorly in the ER lumen due to intermolecular disulfide bond formation, but this problem can be overcome by using superfolder variants $[22,23]$. We chose a monomeric superfolder GFP (msGFP) as a reporter to track passage through the yeast secretory pathway.

The first goal was to determine whether the $\alpha$-factor pro region enhances secretion of msGFP. For this purpose, we fused msGFP either downstream of the $\alpha$-factor signal sequence alone to yield pre- $\alpha \mathrm{f}$-msGFP, or downstream of the entire $\alpha$-factor secretion signal to yield pre-pro- $\alpha \mathrm{f}$ msGFP. These constructs were expressed in S. cerevisiae using the strong constitutive TPI1 promoter. msGFP secretion into the medium was detected by immunoblotting. As shown in Figure 1A, almost no secretion of msGFP was seen with pre- $\alpha \mathrm{f}-\mathrm{msGFP}$, but robust secretion was seen with pre-pro- $\alpha \mathrm{f}-\mathrm{msGFP}$. Subsequent experiments indicated that total expression levels were not significantly affected

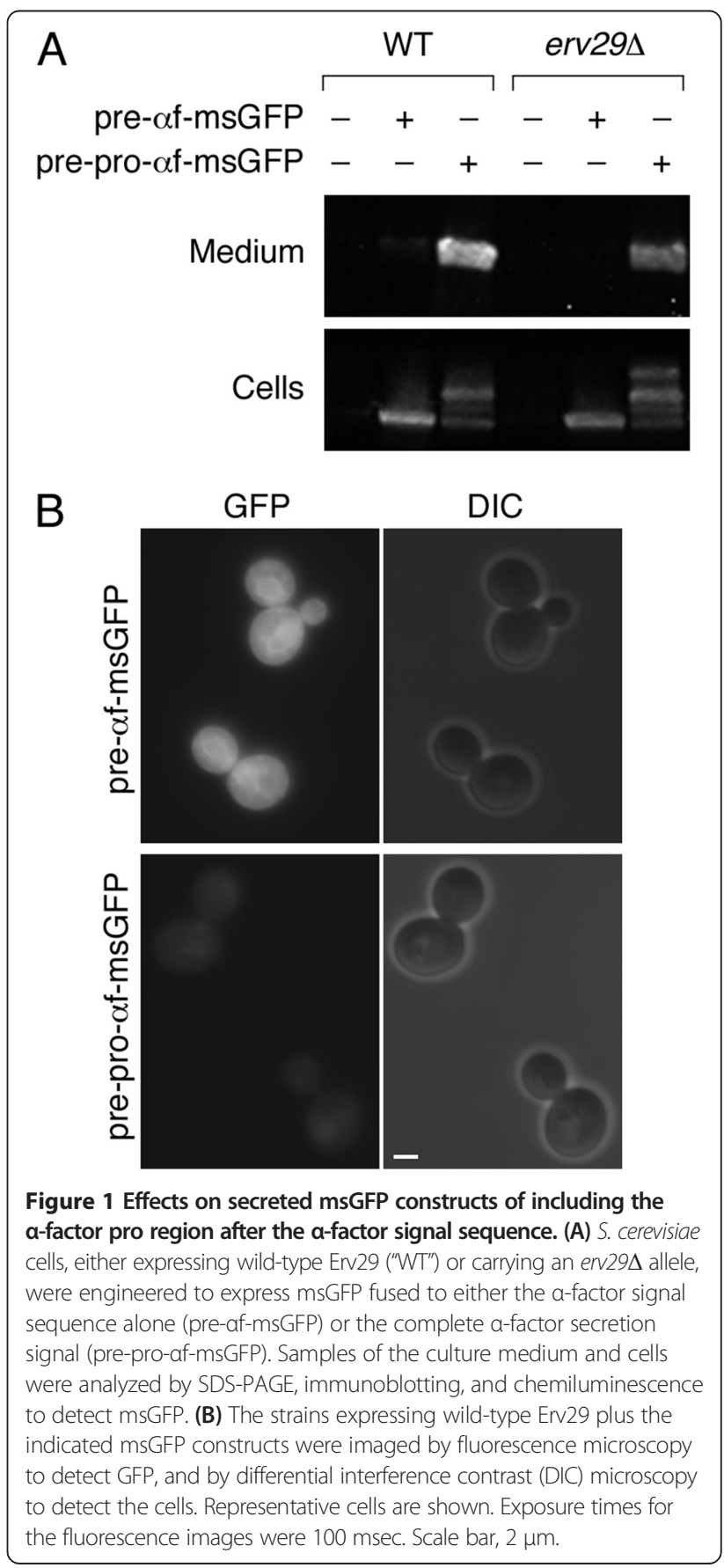


by the presence of the pro region (see Figure 2 below). Thus, secretion of msGFP is stimulated by the $\alpha$-factor pro region.

The only previously characterized function of the $\alpha$ factor pro region is to interact with the ER export receptor Erv29 [24]. We therefore hypothesized that Erv29-dependent acceleration of ER export was responsible for the ability of the pro region to enhance secretion of msGFP. To test this idea, the experiment was repeated with an erv29 strain. This mutation reduced secretion of pre-pro- $\alpha f-m s G F P$, but surprisingly, pre-pro- $\alpha f-m s G F P$ was still secreted much better than pre- $\alpha \mathrm{f}$-msGFP (Figure 1A). This result indicates that the enhancement of secretion by the pro region is largely independent of Erv29.

To identify the roadblock in secretion with the pre- $\alpha f-$ msGFP construct, we examined the cells by fluorescence microscopy. The results were striking: with pre- $\alpha f-m s G F P$, strong cytosolic fluorescence was seen, whereas with pre-pro- $\alpha f-m s G F P$, the cytosolic signal was much weaker (Figure 1B). A likely interpretation is that the pro region facilitates translocation of msGFP across the ER membrane.

\section{The pro region enhances translocation into the ER}

As a direct test of whether pre- $\alpha \mathrm{f}$-msGFP is translocated across the ER membrane less efficiently than pre-pro- $\alpha \mathrm{f}-$ msGFP, we modified the proteins to be retained in the

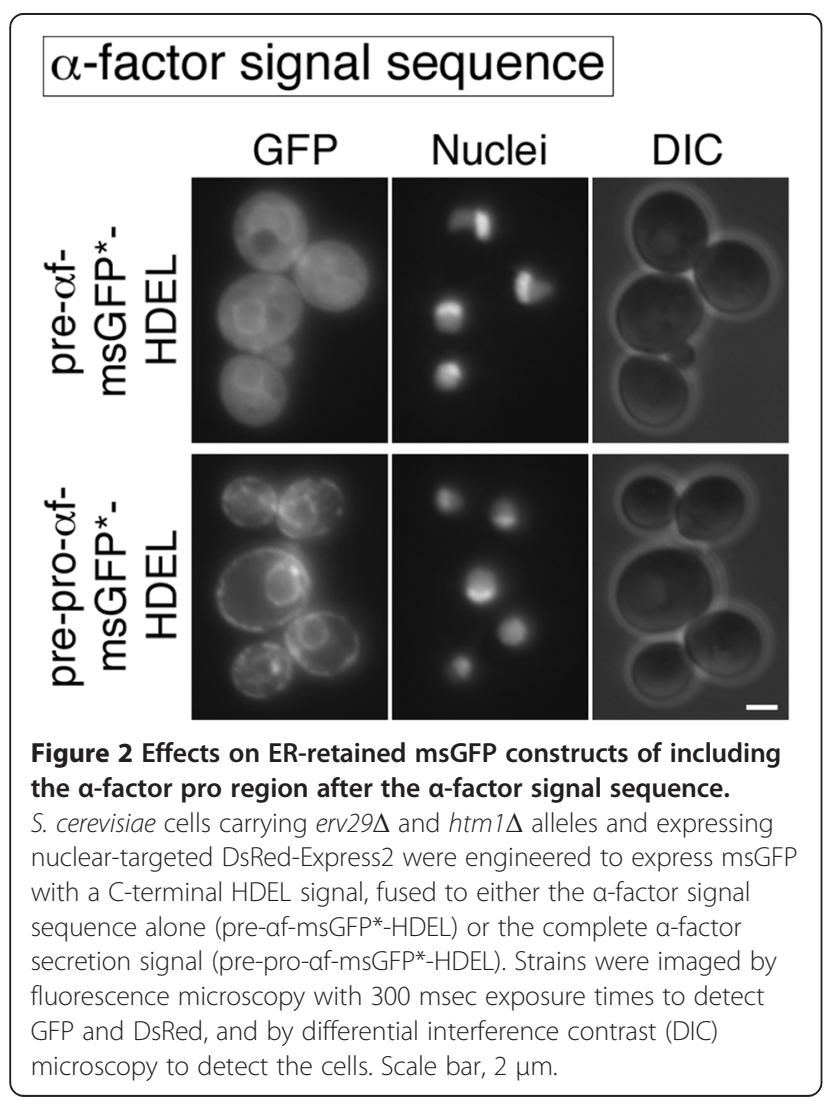

ER by means of a C-terminal HDEL tetrapeptide [25]. Our experience is that residues preceding an HDEL signal can influence its efficacy, so to generate a signal that is known to cause ER retention [26], we also replaced the C-terminal peptide of msGFP with the wild-type GFP C-terminal peptide to create msGFP*-HDEL constructs. Removal of the signal sequence in the ER will yield either msGFP*-HDEL in the case of pre- $\alpha f-m s G F P *-H D E L$, or pro- $\alpha \mathrm{f}-\mathrm{msGFP} *$-HDEL in the case of pre-pro- $\alpha \mathrm{f}-\mathrm{msGFP} *$ HDEL. The latter construct introduces two complications. First, Erv29 will recognize the pro region and direct rapid ER export, thereby potentially compromising ER retention. To avoid this problem, we used an erv294 strain. Second, the three N-linked oligosaccharides in the pro region [27] will eventually be processed by $\mathrm{Htm} 1$ to trigger ER-associated degradation [28]. To avoid this problem, we introduced the $h t m 1 \Delta$ mutation. Finally, to identify the ER, which includes a prominent nuclear envelope ring [29-31], we integrated a construct for expressing DsRed-Express2 fused to a nuclear localization signal [32].

The pre- $\alpha \mathrm{f}$-msGFP*-HDEL construct generated a strong cytosolic signal plus a weak ER signal (Figure 2). By contrast, the pre-pro- $\alpha \mathrm{f}-\mathrm{msGFP} *$-HDEL construct generated a weak cytosolic signal plus a strong ER signal (Figure 2). This result confirms that inclusion of the pro region downstream of the $\alpha$-factor signal sequence stimulates translocation of msGFP into the ER. We infer that posttranslational translocation of msGFP is inefficient with the $\alpha$-factor signal sequence alone, and that inclusion of the pro region increases the amount of msGFP that ultimately crosses the ER membrane.

The cotranslational Ost1 signal sequence bypasses the need for the pro region

For msGFP secretion, an alternative signal sequence that directs cotranslational translocation might be more effective than the $\alpha$-factor signal sequence. Cleavable signal sequences in yeast typically direct posttranslational translocation [12,33], but an exception is the $S$. cerevisiae Ost1 signal sequence, which has been shown to direct cotranslational translocation [14,34]. We therefore repeated the msGFP*-HDEL experiment after replacing the $\alpha$-factor signal sequence with the Ost1 signal sequence. As shown in Figure 3, both pre-Ost1-msGFP*-HDEL and pre-Ost1-pro- $\alpha$-msGFP*-HDEL generated fluorescent ER compartments with little cytosolic background. Thus, for targeting msGFP to the secretory pathway, the cotranslational Ost1 signal sequence bypasses the requirement for the $\alpha$-factor pro region.

To verify this conclusion, we examined secretion of msGFP with the Ost1 signal sequence. As shown in Figure 4A, for wild-type or erv294 cells, the amount of msGFP in the culture medium with pre-Ost1-msGFP 


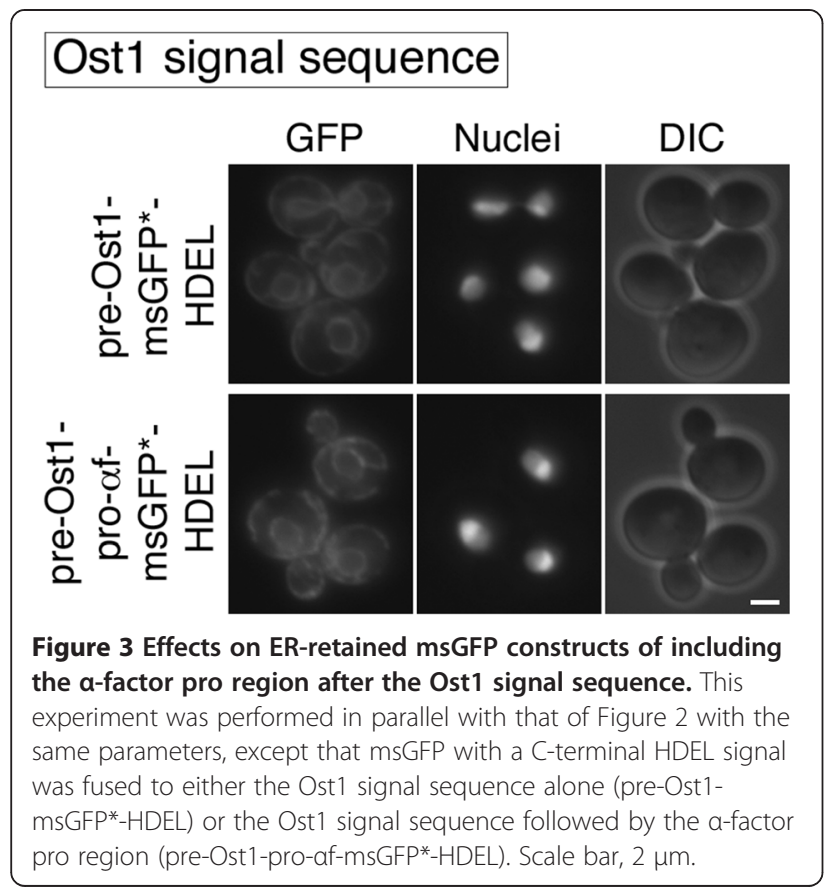

was at least as high as the amount with pre-pro- $\alpha$ msGFP. Inclusion of the $\alpha$-factor pro region after the Ost1 signal sequence to create pre-Ost1-pro- $\alpha \mathrm{f}$-msGFP did not enhance secretion, and was actually inhibitory (Figure 4A). These results indicate that for msGFP, cotranslational translocation with the Ost1 signal sequence alone is sufficient for secretion.

Additional insight came from fluorescence microscopy of strains expressing the secreted msGFP constructs. pre-Ost1-msGFP generated fluorescent nuclear envelope and cortical structures typical for the ER, as well as large solid fluorescence signals that corresponded to the vacuolar lumen (Figure 4B). We infer that cotranslational translocation of pre-Ost1-msGFP resulted in delivery to the ER lumen, and that some of the msGFP molecules were then targeted to the vacuole. The pattern was different with pre-pro- $\alpha f-m s G F P$ and pre-Ost1-pro- $\alpha f-m s G F P$, which generated only vacuolar fluorescence (Figure 4B), presumably because Erv29 recognized the pro region and mediated rapid ER export. Indeed, in an erv294 strain, pre-pro- $\alpha \mathrm{f}-\mathrm{msGFP}$ and pre-Ost1-pro- $\alpha \mathrm{f}-\mathrm{msGFP}$ showed not only a vacuolar signal, but also an ER signal (Figure 4C). ER-localized msGFP molecules that retained the pro region probably account for a highmolecular weight species detected in erv294 cells but not in wild-type cells (Figure 4A, asterisk). In sum, the Ost1 signal sequence confers efficient translocation of msGFP into the ER, and inclusion of the $\alpha$-factor pro region confers rapid Erv29-dependent ER exit, but rapid ER exit does not enhance secretion under our experimental conditions.

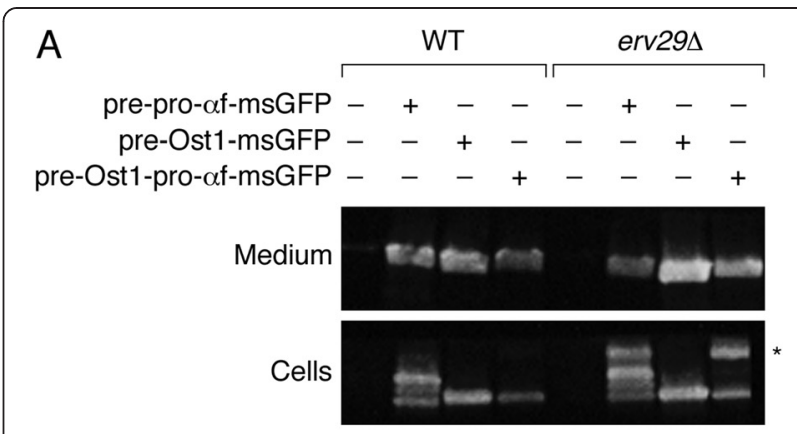

B WT

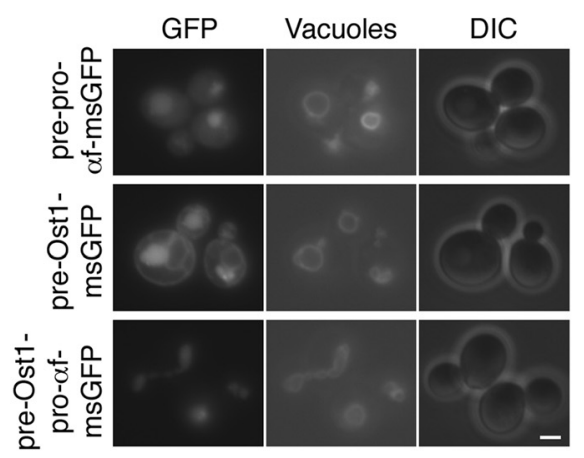

\section{C erv29D}

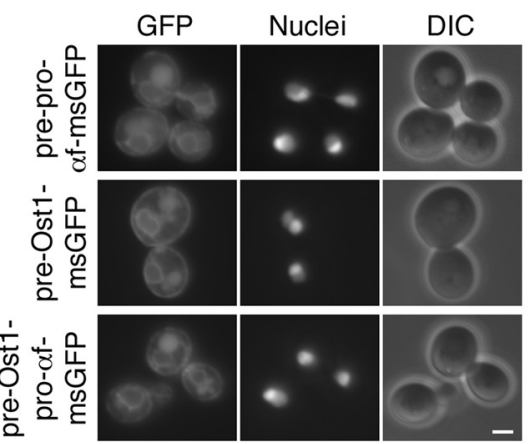

Figure 4 Effects on secreted msGFP constructs of including the a-factor pro region after the Ost1 signal sequence. (A) The analysis was performed as in Figure 1A, except that msGFP was fused to either the complete a-factor secretion signal (pre-pro-af-msGFP), or the Ost1 signal sequence alone (pre-Ost1-msGFP), or the Ost1 signal sequence followed by the a-factor pro region (pre-Ost1-pro-af-msGFP). The asterisk marks a band that may represent ER-localized msGFP molecules fused to the pro region. (B) The analysis was performed as in Figure 1B, except with the strains described in (A). Cells were stained with FM 4-64 to visualize the vacuolar membrane. Exposure times for the fluorescence images were $300 \mathrm{msec}$. Scale bar, 2 $\mu \mathrm{m}$. (C) The analysis was performed as in (B), except that vacuoles were not labeled, and the cells carried an erv29 $\Delta$ allele and expressed nuclear-targeted DsRed-Express2. Exposure times for the fluorescence images were $300 \mathrm{msec}$. Scale bar, 2 $\mu \mathrm{m}$. 


\section{A partial deletion of Vps10 prevents vacuolar targeting of msGFP}

The vacuolar accumulation of msGFP resembles previous observations that GFP variants were targeted to the yeast vacuole [21,35]. Intriguingly, vacuolar targeting of GFP was shown to depend on the sorting receptor Vps10 [21]. We therefore engineered a vps10 $\Delta$ strain, and then repeated the fluorescence microscopy analysis with preOst1-msGFP (Figure 5A). The vacuolar signal was virtually abolished by the vps $10 \Delta$ mutation, leaving only an ER signal. This result confirms that Vps10 can recognize folded GFP for delivery to the vacuole.

The N-terminal lumenal portion of Vps10 consists of two related domains termed domains 1 and 2, and selective removal of domain 1 to create the vps10-104 mutation reportedly preserved the localization, stability, and vacuolar hydrolase sorting capacity of Vps10 [15]. No ligands for domain 1 have been described. We speculated that domain 1 might recognize protein structures that are not normally present in the yeast secretory pathway, in which case removal of domain 1 with the vps10-104 mutation might prevent targeting of msGFP to the vacuole while permitting correct sorting of vacuolar hydrolases. Indeed, in a vps10-104 strain, pre-Ost1-msGFP generated an ER signal but no vacuolar signal, a pattern indistinguishable from that seen in the vps10 $\Delta$ strain (Figure 5A). Immunoblotting of intracellular and secreted carboxypeptidase Y (CPY), which is a ligand for Vps10, confirmed that CPY was missorted to the culture medium in the vps $10 \Delta$ strain but was largely retained intracellularly in the vps10-104 strain (Figure 5B) [17,20]. Thus, a selective mutation in Vps10 can suppress targeting of a foreign protein to the vacuole while preserving other aspects of vacuolar function.

Elimination of vacuolar targeting would be expected to increase the secretion of msGFP. We compared the secretion of msGFP from wild-type, vps10 , and vps10-104 strains expressing either pre-Ost1-msGFP or pre-Ost1pro- $\alpha \mathrm{f}-\mathrm{msGFP}$ (Figure 5C). The vps10 $\Delta$ and vps10-104 mutations boosted secretion $\sim 2.5$-fold for pre-Ost1-msGFP
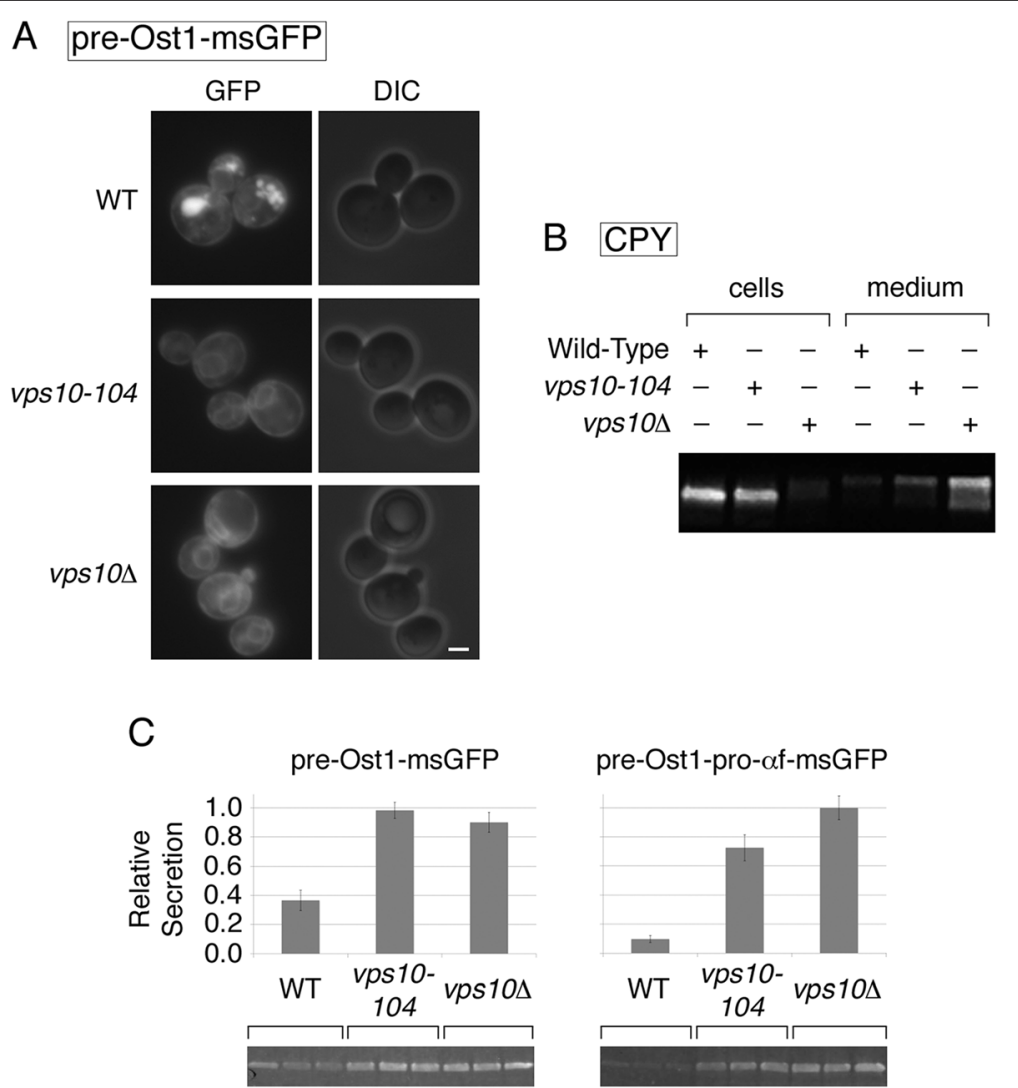

Figure 5 Effects on secreted msGFP constructs of deleting or mutating Vps10. (A) S. cerevisiae cells, either expressing wild-type Vps10 ("WT") or carrying a vps10-104 or vps10 $\Delta$ allele, were engineered to express msGFP fused to the Ost1 signal sequence (pre-Ost1-msGFP). Exposure times for the fluorescence images were $500 \mathrm{msec}$. Scale bar, 2 m. (B) Intracellular and extracellular CPY for the indicated strains was analyzed by SDS-PAGE and immunoblotting as described in Methods. (C) msGFP secretion was analyzed as in Figure $1 \mathrm{~A}$ for the indicated strains, except that immunoblotting was performed with the quantitative procedure described in Methods. The experiment was done twice, with three replicates each time. Immunoblots from one of the experiments are shown. Error bars represent s.e.m. 
and $\sim 8$-fold for pre-Ost1-pro- $\alpha$ f-msGFP. These results confirm that suppression of vacuolar targeting increased the secretion efficiency of a model protein.

\section{Translocation into the ER can be a roadblock in secretion with Pichia pastoris}

As an indication of whether this analysis can be generalized to other budding yeasts, we expressed different msGFP constructs in $P$. pastoris. Cells were transformed with integrating vectors that used the methanol-inducible $A O X 1$ promoter [36,37] to express pre- $\alpha f-m s G F P$, or pre-pro- $\alpha f-$ msGFP, or pre-Ost1-msGFP, or pre-Ost1-pro- $\alpha f-m s G F P$. Secretion of msGFP was weak with the $\alpha$-factor signal sequence alone, and was stronger with the complete $\alpha$ factor secretion signal (Figure 6). Even stronger secretion was seen with the Ost1 signal sequence, either alone or followed by the $\alpha$-factor pro region (Figure 6). The hybrid secretion signal consisting of the Ost1 signal sequence plus the $\alpha$-factor pro region yielded robust secretion of msGFP with minimal accumulation in the cells. Fluorescence microscopy revealed that expression of pre- $\alpha$ fmsGFP generated mainly cytosolic fluorescence, whereas expression of pre-Ost1-msGFP generated ER fluorescence (Figure 7). Very little cellular fluorescence was seen with the pre-pro- $\alpha \mathrm{f}$-msGFP construct, and even less with the pre-Ost1-pro- $\alpha \mathrm{f}-\mathrm{ms}$ GFP contruct (Figure 7). P. pastoris showed negligible vacuolar fluorescence with the msGFP constructs, perhaps because P. pastoris Vps10 has a low affinity for msGFP, but otherwise these results are similar to those seen with S. cerevisiae.

The data obtained with $P$. pastoris support three conclusions about msGFP: (1) Cotranslational translocation driven by the Ost1 signal sequence was more efficient

\begin{tabular}{|c|c|c|c|c|}
\hline pre- $\alpha f-m s G F P$ & + & - & - & - \\
\hline pre-pro- $\alpha f-m s G F P$ & - & + & - & - \\
\hline pre-Ost1-msGFP & - & - & + & - \\
\hline pre-Ost1-pro- $\alpha$ f-msGFP & - & - & - & + \\
\hline
\end{tabular}

Medium

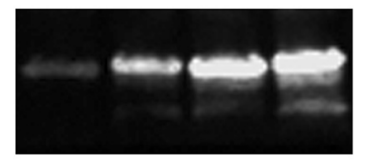

Cells

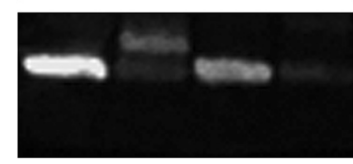

Figure 6 Effects of the different signal sequences on secretion of msGFP from $P$. pastoris. Strains of $P$. pastoris were engineered to express msGFP fused to either the a factor signal sequence alone (preaf-msGFP), or the complete a factor secretion signal (pre-pro-af-msGFP), or the Ost1 signal sequence alone (pre-Ost1-msGFP), or the Ost1 signal sequence followed by the a factor pro region (pre-Ost1-pro-af-msGFP). Extracellular and intracellular msGFP was analyzed as in Figure 1A.

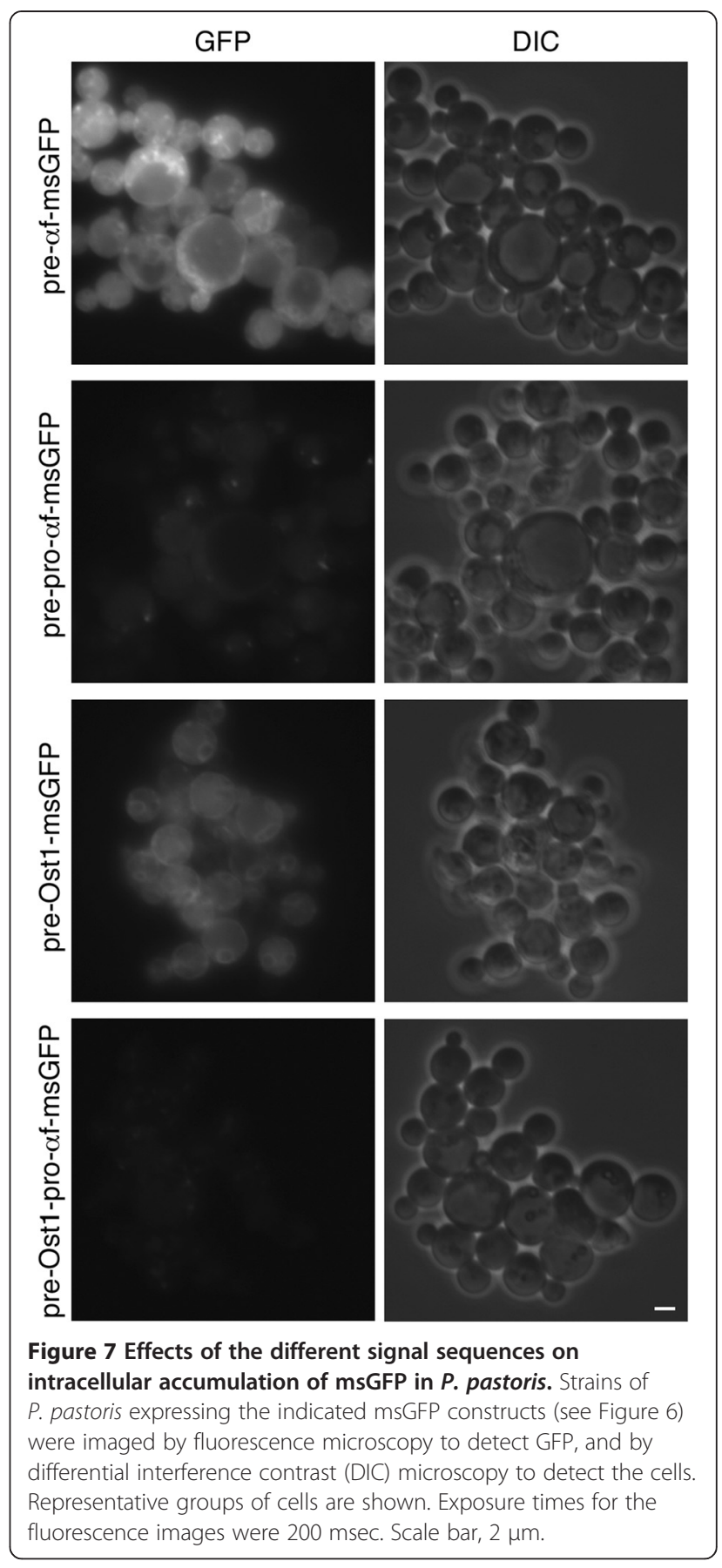

than posttranslational translocation driven by the $\alpha$-factor signal sequence. (2) The $\alpha$-factor pro region stimulated posttranslational translocation when fused downstream of the $\alpha$-factor signal sequence. (3) The $\alpha$-factor pro region stimulated ER export when fused downstream of the signal sequence from either $\alpha$-factor or Ost1.

\section{Discussion}

One motivation for studying the yeast secretory pathway is to engineer this system for improved secretion of 
foreign proteins. Here, we used msGFP as a model secretory protein, reasoning that fluorescence would enable us to visualize roadblocks that are hard to detect by other means. Our analysis employed standard tools of cell biological analysis. With luck, approaches that led to better msGFP secretion under these laboratory conditions will be applicable to other foreign proteins under industrial conditions.

We began by testing the $\alpha$-factor secretion signal, consisting of the signal sequence plus pro region, because wild-type and mutant versions of this signal are commonly used to secrete foreign proteins $[1,2,38,39]$. Inclusion of the $\alpha$-factor pro region was reportedly needed for high-level secretion of human insulin-like growth factor 1 (IGF1) from S. cerevisiae [40] and of human lysozyme from $P$. pastoris [9]. Our experiments with msGFP fusion proteins expressed in S. cerevisiae and P. pastoris revealed that the $\alpha$-factor signal sequence alone yielded minimal secretion, while the full-length $\alpha$-factor secretion signal yielded efficient secretion. Thus, the $\alpha$-factor pro region has repeatedly been found to stimulate secretion, but the mechanistic basis for this effect has been unclear.

One possibility is that the pro region enhances secretion by accelerating export from the ER. Many proteins, including pre-pro- $\alpha$-factor, leave the ER by receptor-dependent pathways $[41,42]$. ER export of pre-pro- $\alpha$-factor involves recognition of the pro region by the transmembrane receptor Erv29 [24,41]. However, we found that with an erv294 strain of $S$. cerevisiae, the full-length $\alpha$-factor secretion signal was still much more effective than the $\alpha$-factor secretion signal alone at driving secretion. This result pointed to a different explanation for the ability of the pro region to enhance secretion of msGFP.

Our attention then turned to translocation, because pre-pro- $\alpha$-factor crosses the ER membrane in a posttranslational manner, and fusion proteins with the $\alpha$ factor signal sequence presumably follow the same pathway $[12,13,43]$. Posttranslational translocation into the ER is thought to employ a Brownian ratchet, in which the polypeptide chain slides back and forth in the translocon and is captured in the lumen by binding of the Hsp70 chaperone Kar2 (BiP) [44]. Posttranslational translocation can be slowed or blocked by the presence of a folded domain in the cytosol [45]. Based on the folding pathway of GFP [46], we speculate that initial folding of immature msGFP impedes translocation, and that more stable folding upon chromophore maturation blocks translocation entirely.

According to this hypothesis, the $\alpha$-factor pro region enhances secretion of msGFP by stimulating translocation into the ER. Our fluorescence microscopy data favor this idea. When msGFP was fused to the $\alpha$-factor signal sequence alone, fluorescent msGFP accumulated in the cytosol, but when msGFP was fused to the full-length $\alpha$ - factor secretion signal, much less cytosolic fluorescence was seen. To focus specifically on the ER translocation step, we appended an HDEL retention signal to msGFP. As expected, fusion of the $\alpha$-factor signal sequence alone to HDEL-tagged msGFP generated mainly cytosolic fluorescence, whereas fusion of the full-length $\alpha$-factor secretion signal to HDEL-tagged msGFP generated ER fluorescence. These findings are reminiscent of an earlier report that with $S$. cerevisiae, fusion of the $\alpha$-factor signal sequence or two other signal sequences to IGF1 resulted in intracellular accumulation, whereas fusion of the full-length $\alpha$-factor secretion signal to IGF1 resulted in secretion [40]. Therefore, inclusion of the $\alpha$-factor pro region apparently enables msGFP and other foreign proteins to enter the yeast ER.

How does the pro region stimulate translocation? The answer is unknown, but the pro region may simply provide a long stretch of unfolded polypeptide upstream of the folded passenger protein, thereby allowing Kar2 to act as an effective ratchet. The three $\mathrm{N}$-linked oligosaccharides that are added to the pro region [27] may further promote directional translocation by hindering backsliding of the polypeptide chain in the translocon.

This analysis prompted us to test a cotranslational translocation pathway, which would permit msGFP to cross the ER membrane prior to folding [45]. Cotranslational translocation into the yeast ER occurs when the signal sequence is sufficiently hydrophobic [12,13]. Only a small fraction of cleavable yeast signal sequences meet this criterion [33], but the hydrophobic Ost1 signal sequence has been rigorously shown to direct cotranslational translocation [14,34]. When fused to msGFP, the Ost1 signal sequence yielded efficient translocation as well as efficient secretion. Inclusion of the $\alpha$-factor pro region after the Ost1 signal sequence did not stimulate translocation. These results confirm that with msGFP, the requirement for the pro region can be bypassed by using a signal sequence that directs cotranslational translocation.

We propose that the Ost1 signal sequence, or similar hydrophobic signal sequences, may prove generally useful for secreting foreign proteins. Compared to the $\alpha$ factor secretion signal, the Ost1 signal sequence has two advantages. First, because translocation with the Ost1 signal sequence is cotranslational, even rapidly folding proteins should enter the ER efficiently. By contrast, with the $\alpha$-factor secretion signal, some rapidly folding proteins might be trapped in the cytosol despite the presence of the pro region. Second, because the Ost1 signal sequence requires cleavage only by signal peptidase, the N-termini of the mature proteins are likely to be homogeneous. By contrast, with the $\alpha$-factor secretion signal, proteolytic removal of the pro region or the downstream EAEA peptide is often incomplete. On the other hand, a potential concern with the Ost1 signal sequence is 
that when fusion proteins are expressed at very high levels, the cotranslational targeting pathway might be overwhelmed. Empirical tests will be needed to assess the practical value of the Ost1 signal sequence.

A foreign protein that crosses the ER membrane must then be exported to the Golgi. As described above, ER export can be accelerated by receptors such as Erv29, which recognizes the $\alpha$-factor pro region. In both $S$. cerevisiae and P. pastoris, we saw accumulation of msGFP in the ER with the Ost1 signal sequence alone, but not with the Ost1 signal sequence followed by the $\alpha$-factor pro region, suggesting that the pro region accelerated ER export. This effect of the pro region was abolished in S. cerevisiae by deleting Erv29. Yet inclusion of the pro region after the Ost1 signal sequence did not enhance secretion of msGFP, presumably because receptorindependent "bulk flow" export from the ER is quite efficient [47]. It is possible that at very high expression levels, Erv29-dependent ER export could aid secretion by preventing excess protein accumulation in the ER, in which case a hybrid secretion signal consisting of the Ost1 signal sequence followed by the $\alpha$-factor pro region may prove to be useful.

Another potential roadblock in secretion occurs during export from the Golgi. Yeast cells have a quality control system in which the vacuolar sorting receptor Vps10 targets misfolded proteins from the Golgi to the vacuole $[15,18,19]$. Vps10 can also recognize folded proteins such as GFP and $\alpha-1$ antitrypsin [21,48], suggesting that Vps10 acts broadly to capture proteins that display conformations not normally found in the secretory pathway. The implication is that for some foreign proteins, deleting Vps10 might enhance secretion by preventing vacuolar targeting. Indeed, strains lacking a Vps10 homolog showed improved secretion in P. pastoris [49], and similar results were seen with the fission yeast Schizosaccharomyces pombe and the filamentous fungus Aspergillus oryzae [50,51]. In our studies of S. cerevisiae, msGFP accumulated in the vacuole, and this vacuolar accumulation was prevented by deleting Vps10.

A problem with deleting Vps10 is that CPY and some other vacuolar hydrolases will be missorted to the culture medium $[15-17,20]$. These secreted vacuolar hydrolases will contaminate a secreted foreign protein and may contribute to its degradation. Ideally, Vps10 would be mutated so that it continues to sort vacuolar hydrolases while no longer targeting foreign proteins to the vacuole.

To engineer such a mutation in Vps10, we built on earlier structure-function studies of the Vps10 family, which includes mammalian sortilin [52]. Fungal members of this family contain two lumenal domains termed domains 1 and 2, each of which is related to the single domain in sortilin [15]. The sortilin domain consists mainly of a tenbladed $\beta$-propeller with a central tunnel [53]. Sequence alignment revealed that sortilin is more similar to domain 2 of S. cerevisiae Vps10 than to domain 1 [15]. In support of this conclusion, structure predictions obtained with the Phyre server [54] indicate that domain 2 of Vps10 is likely to resemble the sortilin domain, whereas domain 1 may have a somewhat modified fold that obscures the central tunnel (Figure 8). Previous studies of S. cerevisiae demonstrated that vacuolar hydrolases are sorted by domain 2 , and that domain 1 could be removed with no significant effect on vacuolar function [15]. Based on these findings, we speculated that domain 2 of yeast Vps10 has a conserved role in sorting vacuolar hydrolases, while domain 1 might be a fungal-specific adaptation for recognizing abnormal protein structures.

To test this hypothesis, we replaced S. cerevisiae Vps10 with a truncated version lacking domain 1 [15]. The strain with truncated Vps10 sorted CPY almost normally, but like the vps10 1 strain, it did not accumulate msGFP in the vacuole. This result establishes msGFP as the first known ligand for domain 1 of Vps10. Truncation of Vps10 enhanced the secretion of msGFP from $S$. cerevisiae, consistent with a higher percentage of the msGFP molecules trafficking from the Golgi to the cell surface. It remains to be determined whether other folded foreign proteins are also recognized by domain 1 . If so, then truncation of Vps10 is an attractive option for boosting the secretion of foreign proteins from budding yeasts, and potentially from other fungi, without substantially altering vacuolar function.

Our experiments show that cell biological studies with a model protein can suggest avenues for increasing the secretion of foreign proteins from yeasts. If a protein is poorly secreted or incompletely processed with existing approaches, we offer two recommendations. First, the Ost1 signal sequence drives efficient translocation into the ER, and avoids the incomplete processing caused by the $\alpha$-factor pro region. If incomplete processing is not a concern, a hybrid secretion signal consisting of the Ost1 signal sequence followed by the $\alpha$-factor pro region might be beneficial for ensuring both efficient translocation and efficient ER export. Second, selective removal of domain 1 of Vps10 can prevent a foreign protein from being diverted to the vacuole. It will be interesting to test these approaches with commercially and therapeutically relevant foreign proteins.

\section{Methods}

\section{Strains and plasmids}

S. cerevisiae strains were derivatives of the haploid strain JK9-3da (leu2-3,112 ura3-52 rme1 trp1 his4) [55]. These cells were grown in rich glucose medium (YPD) supplemented with $20 \mu \mathrm{g} / \mathrm{mL}$ each of adenine and uracil, or in minimal glucose dropout medium (SD) [56] prepared using nutrient mixtures from Sunrise Science Products. 

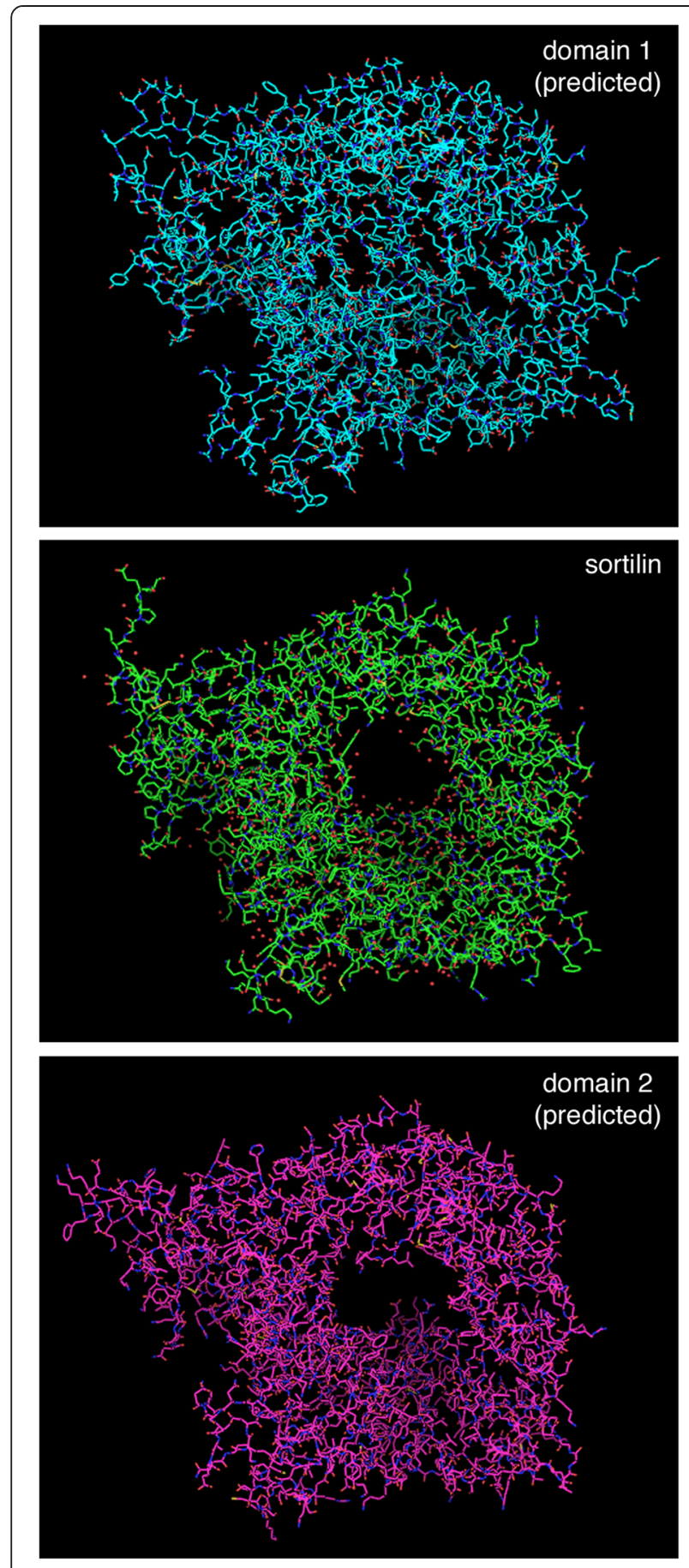

Figure 8 Predicted structures of domains 1 and 2 of Vps 10 compared to the known structure of sortilin. The protein sequences of S. cerevisiae Vps 10 domain 1 (residues 22-737) and domain 2 (residues 719-1393) were submitted to the protein homology/analogy recognition engine Phyre (http://www.sbg.bio.ic.ac. uk/phyre/html/) [54], which detected the similarity to sortilin and generated PDB files for the predicted tertiary structures. A file for the experimentally determined structure of the human sortilin lumenal domain (PDB ID 3F6K) [53] was downloaded from the National Center for Biotechnology Information. To generate the images shown, these PDB files were opened with MacPyMOL using the default settings.
P. pastoris strains were derivatives of the haploid strain PPY12 (his4 arg4) [57]. These cells were grown as precultures in YPD, then transferred to minimal glycerol medium containing $0.05 \%$ yeast extract (SYG), then transferred to minimal methanol medium containing $0.05 \%$ yeast extract (SYM) [37]. All yeast cultures were grown with shaking at $200 \mathrm{rpm}$ in baffled flasks.

Molecular biology procedures were simulated and recorded using SnapGene software (GSL Biotech), and the supplementary information includes a folder of annotated plasmid sequence/map files that can be opened with SnapGene Viewer (http://www.snapgene.com/products/ snapgene_viewer/) [see Additional file 1]. msGFP was derived from enhanced GFP by introducing superfolder mutations [23], as well as the monomerizing A206K mutation [58] plus modified $\mathrm{N}$ - and C-terminal peptides. A full characterization of msGFP will be provided elsewhere (manuscript in preparation), but the sequence of msGFP is available in the supplementary SnapGene files. For constitutive expression in $S$. cerevisiae, msGFP was fused to the $\alpha$-factor or Ost1 signal sequence, with or without the $\alpha$-factor pro region. For retention in the ER, the C-terminal peptide of msGFP was replaced with the wild-type GFP C-terminal peptide plus HDEL [26]. Constructs were expressed using the TPI1 promoter and CYC1 terminator in the TRP1 integrating vector YIplac204 [59]. For regulated expression in P. pastoris, msGFP constructs were transferred into the HIS4 integrating vector pIB4, which contains the methanol-inducible $A O X 1$ promoter [37]. Integrating vectors were linearized with restriction enzymes as indicated in the supplementary SnapGene files, and were then transformed into $S$. cerevisiae using lithium acetate [60] or into P. pastoris using electroporation [37]. Transformants were selected on minimal dropout plates, with histidine, leucine, or tryptophan omitted as needed to select for integration of the linearized vectors. To avoid multi-copy integrants, a number of clones from each transformation were screened by fluorescence microscopy, and clones with unusually high fluorescence signals were excluded from further analysis.

Gene deletions were performed using standard methods. Briefly, to delete the S. cerevisiae ERV29 gene, the kanMX cassette was amplified from pFA6a-kanMX6 [61] using primers GACTCAAAAAAAGTGAAAACAAAACTGAA AGGATAGATCACGTACGCTGCAGGTCGAC and GAG TGAACAGAAGGGACATAAAGAAAAGATTTCCTTTA CAATATCGATGAATTCGAGCTCG. The resulting fragment was transformed into cells, which were plated on YPD medium containing $250 \mu \mathrm{g} / \mathrm{ml} \mathrm{G418} \mathrm{(Sigma-Aldrich)}$ to select for double-crossover replacement of the ERV29 open reading frame. A similar approach was used to delete the $S$. cerevisiae HTM1 gene, except that a hygromycin resistance cassette was amplified from pAG32 [62] using primers GAGTAACCATGATAATTTCATATTTCCATG 
GATTGGTTACATTCAGGGAAATAGACCAGATCTGT TTAGCTTGCCTTGTCC and CATTTATTACTGGT GCCATTATGTAAAAGCTGTAGAGGTCTATCTAAA AGAGTGATTCGTTTTCGACACTGGATGGCGGCG TTAG, and selection was performed on YPD plates containing $200 \mu \mathrm{g} / \mathrm{mL}$ hygromycin. Each gene deletion was confirmed by two separate diagnostic PCR amplifications of genomic DNA.

\section{Assays for secretion of msGFP and CPY}

For S. cerevisiae, secretion was measured in YPD because the recovery of msGFP for immunoblotting was more reliable in rich than in minimal medium. A $5-\mathrm{mL}$ culture in YPD was inoculated from a preculture, and grown overnight at $30^{\circ} \mathrm{C}$ with shaking in a baffled flask to an $\mathrm{OD}_{600}$ of 0.7-0.8. Then $1.75 \mathrm{OD}_{600}$ units were transferred to a $15-\mathrm{mL}$ tube, washed twice with deionized water by centrifugation and resuspension, and resuspended in $5 \mathrm{~mL}$ of fresh YPD to an $\mathrm{OD}_{600}$ of 0.35 . This culture was incubated with shaking at $30^{\circ} \mathrm{C}$ for $3 \mathrm{~h}$, an incubation period that enabled secretion of a detectable amount of msGFP or CPY while the cells remained in mid-log phase. Then $1.6 \mathrm{~mL}$ of culture was transferred to a microcentrifuge tube. When multiple cultures were processed in parallel, $1.6 \mathrm{~mL}$ of the culture with the lowest $\mathrm{OD}_{600}$ was collected, and an equivalent number of $\mathrm{OD}_{600}$ units was collected from each of the other cultures. These volume adjustments were minor because all of the strains grew at similar rates. Each culture was centrifuged at $3000 \times \mathrm{g}$ (5600 rpm) for $5 \mathrm{~min}$ in a microcentrifuge to separate the cells from the secreted proteins.

For $P$. pastoris, cultures were grown in YPD to an $\mathrm{OD}_{600}$ of $\sim 0.3$, then washed with SYG using a bottle-top filter, then resuspended in SYG and grown overnight. The cultures were then washed and transferred to SYM supplemented with $0.25 \mathrm{mg} / \mathrm{mL}$ bovine serum albumin at an $\mathrm{OD}_{600}$ of $\sim 1.2$. After $3 \mathrm{~h}$ in this inducing medium, the cultures were washed and transferred to fresh SYM supplemented with $0.25 \mathrm{mg} / \mathrm{mL}$ bovine serum albumin. After an additional $3 \mathrm{~h}$, the secreted and intracellular proteins were separated as for S. cerevisiae.

To analyze msGFP or CPY in the culture medium [63], $1.5 \mathrm{~mL}$ of supernatant from the final centrifugation was transferred to a fresh tube, and was supplemented with $167 \mu \mathrm{L}$ of $100 \%(\mathrm{w} / \mathrm{v})$ trichloroacetic acid (TCA). This mixture was left on ice for at least $20 \mathrm{~min}$. The tube was then centrifuged at $16,000 \times \mathrm{g}$ (maximum speed) in a microcentrifuge for $15 \mathrm{~min}$ at $4^{\circ} \mathrm{C}$. The supernatant was carefully removed and discarded. Then the precipitated proteins were resuspended in $50 \mu \mathrm{L}$ SDS-PAGE sample buffer supplemented with $50 \mathrm{mM}$ $\mathrm{Na}^{+}$-PIPES, $\mathrm{pH} 7.5$, and $2 \% \beta$-mercaptoethanol. The samples were boiled for $5 \mathrm{~min}$, then centrifuged for $2 \mathrm{~min}$ at $16,000 \times \mathrm{g}$ in a microcentrifuge to remove insoluble material. Twenty $\mu \mathrm{L}$ of the final supernatant was loaded on a gel for SDS-PAGE.

To analyze msGFP or CPY in the cells [63], the pellet from the final centrifugation was resuspended in $1 \mathrm{~mL}$ of deionized water and then centrifuged at $3000 \times \mathrm{g}$, and the water was removed. The pellet was resuspended in $200 \mu \mathrm{L}$ of $5 \%(\mathrm{w} / \mathrm{v}) \mathrm{TCA}$. This mixture was supplemented with $100 \mu \mathrm{L}$ of $0.5 \mathrm{~mm}$ diameter glass beads (BioSpec Products), and was vortexed for $30 \mathrm{sec}$ to break the cells. Eight hundred $\mu \mathrm{L}$ of $5 \%$ TCA was added and mixed. Then $800 \mu \mathrm{L}$ of this solution was transferred to a fresh tube and left on ice for at least $5 \mathrm{~min}$. The sample was centrifuged and processed as described above, except that the precipitated cellular proteins were dissolved in $400 \mu \mathrm{L}$ SDS-PAGE sample buffer.

For immunoblotting, $20 \mu \mathrm{L}$ of each sample was run on a $4-20 \%$ tricine gel (Bio-Rad). The separated proteins were transferred to an Immobilon membrane (Millipore). Subsequent manipulations were performed at room temperature. The membrane was blocked with 5\% nonfat dry milk in TBST, which was Tris-buffered saline (TBS; $50 \mathrm{mM}$ Tris- $\mathrm{HCl}, \mathrm{pH} 7.6,150 \mathrm{mM} \mathrm{NaCl}$ ) plus $0.05 \%$ Tween 20, and was then incubated with shaking for $1 \mathrm{~h}$ with either 1:1000 goat polyclonal anti-GFP antibody (Applied Biological Materials) or $0.25 \mu \mathrm{g} / \mathrm{mL}$ mouse monoclonal anti-CPY antibody (Invitrogen; clone 10A5B5) in 1\% milk/TBST. After a rinse with 5\% milk/TBST, the membrane was incubated with shaking for $1 \mathrm{~h}$ with a 1:20,000 peroxidase-conjugated secondary antibody in $0.5 \%$ milk/ TBST, either rabbit anti-goat IgG (Sigma-Aldrich) for detecting GFP or sheep anti-mouse IgG (GE Healthcare) for detecting CPY. After final rinses with $5 \%$ milk/TBST followed by TBS, the antibody-bound proteins were detected by chemiluminescence using SuperSignal West Femto Maximum Sensitivity Substrate (Thermo Scientific). Alternatively, the procedure was modified as follows for quantitative analysis of msGFP secretion. The membrane was blocked with Odyssey Blocking Buffer (LI-COR) plus $0.2 \%$ Tween 20 , and the secondary antibody was IRDye 680RD donkey anti-goat (LI-COR) diluted 1:15,000 in Odyssey Blocking Buffer $+0.2 \%$ Tween $20+0.01 \%$ SDS. The signal was detected using a LI-COR Odyssey CLx infrared imaging system. Lanes were specified, and bands were either automatically picked or drawn by the user. The median background was subtracted from each band using pixels above and below the band area, and the integrated intensities of the bands were averaged and normalized.

\section{Fluorescence microscopy}

For imaging intracellular msGFP, DsRed-Express2, and FM 4-64, $1.5 \mu \mathrm{L}$ from a culture at an $\mathrm{OD}_{600}$ of $0.4-0.5$ was compressed on a slide beneath a \#1.5 coverslip. The yeast cells were viewed immediately with a Zeiss Axioplan 2 microscope using a 1.4-NA Plan-Apo objective 
and a FITC or Texas Red filter set (Chroma). Singleplane images were captured with a Hamamatsu digital camera using Zeiss AxioVision software. Images were resized and cropped with Adobe Photoshop, but were not otherwise processed.

Labeling of vacuoles with FM 4-64 was performed as previously described [64]. Briefly, cells were incubated with $0.8 \mu \mathrm{M}$ FM 4-64 for $5 \mathrm{~min}$, and the dye was quenched by adding $2 \mu \mathrm{M}$ 4-sulfonato calix[8]arene, sodium salt (Biotium). The internalized dye was then chased for approximately $1 \mathrm{~h}$ to label the vacuolar membrane.

\section{Additional file}

\section{Additional file 1: Compressed folder of plasmid sequence/map}

files. This ZIP archive contains 13 files corresponding to the plasmid constructs used in the study. The annotated sequences and maps are encoded in SnapGene's .dna format. These files can be opened with the free SnapGene Viewer (http://www.snapgene.com/products/ snapgene_viewer/).

\section{Abbreviations}

CPY: Carboxypeptidase Y; DIC: Differential interference contrast; ER: Endoplasmic reticulum; GFP: Green fluorescent protein; IGF1: Insulin-like growth factor 1; msGFP: Monomeric superfolder GFP; SYG: Minimal glycerol medium; SYM: Minimal methanol medium; TBS: Tris-buffered saline; TBST: Tris-buffered saline plus Tween 20; TCA: Trichloroacetic acid; YPD: Rich glucose medium.

\section{Competing interests}

The authors declare that they have no competing interests.

\section{Authors' contributions}

IF performed all of the experimental manipulations, and helped to design the procedures and interpret the results. BSG provided guidance at all stages of the project, and oversaw the writing of the manuscript. Both authors read and approved the final manuscript.

\section{Acknowledgments}

Thanks to the Glick lab for helpful discussion, to Maya Schuldiner for a protocol to detect secreted GFP, and to S. Andrei Anghel for generating the erv29D strain. This work was supported by NIH grant R01 GM104010 to B.S.G., and by NIH training grant T32 EB009412 to I.F.

\section{Author details}

'Graduate Program in Biophysical Sciences, University of Chicago, 929 East 57th Street, Chicago, Illinois 60637, USA. ${ }^{2}$ Department of Molecular Genetics and Cell Biology, University of Chicago, 920 East 58th Street, Chicago, Illinois 60637, USA.

Received: 25 June 2014 Accepted: 17 August 2014

Published online: 28 August 2014

\section{References}

1. Gasser B, Prielhofer R, Marx H, Maurer M, Nocon J, Steiger M, Puxbaum V, Sauer M, Mattanovich D: Pichia pastoris: protein production host and model organism for biomedical research. Future Microbiol 2013, 8:191-208.

2. Hou J, Tyo KE, Liu Z, Petranovic D, Nielsen J: Metabolic engineering of recombinant protein secretion by Saccharomyces cerevisiae. FEMS Yeast Res 2012, 12:491-510.

3. Idiris A, Tohda $\mathrm{H}$, Kumagai $\mathrm{H}$, Takegawa $\mathrm{K}$ : Engineering of protein secretion in yeast: strategies and impact on protein production. Appl Microbiol Biotechnol 2010, 86:403-417.

4. Johnson EA: Biotechnology of non-Saccharomyces yeasts-the ascomycetes. Appl Microbiol Biotechnol 2013, 97:503-517.
5. Lin Cereghino J, Cregg JM: Heterologous protein expression in the methylotrophic yeast Pichia pastoris. FEMS Microbiol Rev 2000, 24:45-66.

6. Barlowe CK, Miller EA: Secretory protein biogenesis and traffic in the early secretory pathway. Genetics 2013, 193:383-410.

7. Duden R, Schekman R: Insights into Golgi function through mutants in yeast and animal cells. In The Golgi Apparatus. Edited by Berger EG, Roth J. Basel: Birkhäuser Verlag; 1997:219-246.

8. Brake AJ, Merryweather JP, Coit DG, Heberlein UA, Masiarz FR, Mullenbach GT, Urdea MS, Vaenzuela P, Barr PJ: a-Factor-directed synthesis and secretion of mature foreign proteins in Saccharomyces cerevisiae. Proc Natl Acad Sci U S A 1984, 81:4642-4646.

9. Oka C, Tanaka M, Muraki M, Harata K, Suzuki K, Jigami Y: Human lysozyme secretion increased by alpha-factor pro-sequence in Pichia pastoris. Biosci Biotechnol Biochem 1999, 63:1977-1983.

10. Fuller RS, Sterne RE, Thorner J: Enzymes required for yeast prohormone processing. Annu Rev Physiol 1988, 50:345-362.

11. Julius D, Blair L, Brake A, Sprague G, Thorner J: Yeast a factor is processed from a larger precursor polypeptide: the essential role of a membrane-bound dipeptidyl aminopeptidase. Cell 1983, 32:839-852.

12. Ng DT, Brown JD, Walter P: Signal sequences specify the targeting route to the endoplasmic reticulum membrane. J Cell Biol 1996, 134:269-278.

13. Plath K, Mothes W, Wilkinson BM, Stirling CJ, Rapoport TA: Signal sequence recognition in posttranslational protein transport across the yeast ER membrane. Cell 1998, 94:795-807.

14. Willer M, Forte GM, Stirling CJ: Sec61p is required for ERAD-L: genetic dissection of the translocation and ERAD-L functions of Sec61p using novel derivatives of CPY. J Biol Chem 2008, 283:33883-33888.

15. Jørgensen MU, Emr SD, Winther JR: Ligand recognition and domain structure of Vps10p, a vacuolar protein sorting receptor in Saccharomyces cerevisiae. Eur J Biochem 1999, 260:461-469.

16. Westphal V, Marcusson EG, Winther JR, Emr SD, van den Hazel HB: Multiple pathways for vacuolar sorting of yeast proteinase A. J Biol Chem 1996, 271:11865-11870.

17. Cooper AA, Stevens TH: Vps10p cycles between the late-Golgi and prevacuolar compartments in its function as the sorting receptor for multiple yeast vacuolar hydrolases. J Cell Biol 1996, 133:529-541.

18. Holkeri H, Makarow M: Different degradation pathways for heterologous glycoproteins in yeast. FEBS Lett 1998, 429:162-166.

19. Hong E, Davidson AR, Kaiser CA: A pathway for targeting soluble misfolded proteins to the yeast vacuole. J Cell Biol 1996, 135:623-633.

20. Marcusson EG, Horazdovsky BF, Lin Cereghino J, Gharakhanian E, Emr SD: The sorting receptor for yeast vacuolar carboxypeptidase $Y$ is encoded by the VPS10 gene. Cell 1994, 77:579-586.

21. Humair D, Hernández Felipe D, Neuhaus JM, Paris N: Demonstration in yeast of the function of BP-80, a putative plant vacuolar sorting receptor. Plant Cell 2001, 13:781-792

22. Aronson DE, Costantini LM, Snapp EL: Superfolder GFP is fluorescent in oxidizing environments when targeted via the Sec translocon. Traffic 2011, 12:543-548.

23. Pédelacq JD, Cabantous S, Tran T, Terwilliger TC, Waldo GS: Engineering and characterization of a superfolder green fluorescent protein. Nat Biotechnol 2006, 24:79-88.

24. Otte S, Barlowe C: Sorting signals can direct receptor-mediated export of soluble proteins into COPII vesicles. Nat Cell Biol 2004, 6:1189-1194.

25. Pelham $\mathrm{H}$ : Sorting and retrieval between the endoplasmic reticulum and Golgi apparatus. Curr Opin Cell Biol 1995, 7:530-535.

26. Rossanese OW, Reinke CA, Bevis BJ, Hammond AT, Sears IB, O'Connor J, Glick BS: A role for actin, Cdc1p and Myo2p in the inheritance of late Golgi elements in Saccharomyces cerevisiae. J Cell Biol 2001, 153:47-61.

27. Caplan S, Green R, Rocco J, Kurjan J: Glycosylation and structure of the yeast MFa1 a-factor precursor is important for efficient transport through the secretory pathway. J Bacteriol 1991, 173:627-635.

28. Clerc S, Hirsch C, Oggier DM, Deprez P, Jakob C, Sommer T, Aebi M: Htm1 protein generates the $\mathrm{N}$-glycan signal for glycoprotein degradation in the endoplasmic reticulum. J Cell Biol 2009, 184:159-172.

29. Fehrenbacher KL, Davis D, Wu M, Boldogh I, Pon LA: Endoplasmic reticulum dynamics, inheritance, and cytoskeletal interactions in budding yeast. Mol Biol Cell 2002, 13:854-865.

30. Prinz WA, Grzyb L, Veenhuis M, Kahana JA, Silver PA, Rapoport TA: Mutants affecting the structure of the cortical endoplasmic reticulum in Saccharomyces cerevisiae. J Cell Biol 2000, 150:461-474. 
31. Rose MD, Misra LM, Vogel JP: KAR2, a karyogamy gene, is the yeast homolog of the mammalian BiP/GRP78 gene. Cell 1989, 57:1211-1221.

32. Strack RL, Strongin DE, Bhattacharyya D, Tao W, Berman A, Broxmeyer HE, Keenan RJ, Glick BS: A noncytotoxic DsRed variant for whole-cell labeling. Nat Methods 2008, 5:955-957.

33. Ast T, Cohen $\mathrm{G}$, Schuldiner M: A network of cytosolic factors targets SRP-independent proteins to the endoplasmic reticulum. Cell 2013, 152:1134-1145.

34. Forte GM, Pool MR, Stirling CJ: N-terminal acetylation inhibits protein targeting to the endoplasmic reticulum. PLOS Biol 2011, 9:e1001073.

35. Kunze I, Hensel G, Adler K, Bernard J, Neubohn B, Nilsson C, Stoltenburg R, Kohlwein SD, Kunze G: The green fluorescent protein targets secretory proteins to the yeast vacuole. Biochim Biophys Acta 1999, 1410:287-298.

36. Cregg JM, Madden KR, Barringer KJ, Thill GP, Stillman CA: Functional characterization of the two alcohol oxidase genes from the yeast Pichia pastoris. Mol Cell Biol 1989, 9:1316-1323.

37. Sears IB, O'Connor J, Rossanese OW, Glick BS: A versatile set of vectors for constitutive and regulated gene expression in Pichia pastoris. Yeast 1998, 14:783-790.

38. Lin-Cereghino GP, Stark CM, Kim D, Chang J, Shaheen N, Poerwanto H, Agari K, Moua P, Low LK, Tran N, Huang AD, Nattestad M, Oshiro KT, Chang JW, Chavan A, Tsai JW, Lin-Cereghino J: The effect of a-mating factor secretion signal mutations on recombinant protein expression in Pichia pastoris. Gene 2013, 519:311-317.

39. Rakestraw JA, Sazinsky SL, Piatesi A, Antipov E, Wittrup KD: Directed evolution of a secretory leader for the improved expression of heterologous proteins and full-length antibodies in Saccharomyces cerevisiae. Biotechnol Bioeng 2009, 103:1192-1201.

40. Chaudhuri B, Steube K, Stephan C: The pro-region of the yeast prepro-afactor is essential for membrane translocation of human insulin-like growth factor 1 in vivo. Eur J Biochem 1992, 206:793-800.

41. Belden WJ, Barlowe C: Role of Erv29p in collecting soluble secretory proteins into ER-derived transport vesicles. Science 2001, 294:1528-1531.

42. Dancourt J, Barlowe C: Protein sorting receptors in the early secretory pathway. Annu Rev Biochem 2010, 79:777-802.

43. Rothblatt JA, Deshaies RJ, Sanders SL, Daum G, Schekman R: Multiple genes are required for proper insertion of secretory proteins into the endoplasmic reticulum in yeast. J Cell Biol 1989, 109:2641-2652.

44. Matlack KE, Misselwitz B, Plath K, Rapoport TA: BiP acts as a molecular ratchet during posttranslational transport of prepro-a factor across the ER membrane. Cell 1999, 97:553-564.

45. Schatz $\mathrm{G}$, Dobberstein B: Common principles of protein translocation across membranes. Science 1996, 271:1519-1526.

46. Craggs TD: Green fluorescent protein: structure, folding and chromophore maturation. Chem Soc Rev 2009, 38:2865-2875.

47. Thor F, Gautschi M, Geiger R, Helenius A: Bulk flow revisited: transport of a soluble protein in the secretory pathway. Traffic 2009, 10:1819-1830.

48. Gelling CL, Dawes IW, Perlmutter DH, Fisher EA, Brodsky JL: The endosomal protein-sorting receptor sortilin has a role in trafficking a 1 antitrypsin. Genetics 2012, 192:889-903.

49. Meehl M, Lin H, Choi BK: In Methods for the production of recombinant proteins with improved secretion efficiencies. Edited by Office UPT. United States: Merck Sharpe \& Dohme Corp; 2013.

50. Idiris A, Tohda H, Sasaki M, Okada K, Kumagai H, Giga-Hama Y, Takegawa K: Enhanced protein secretion from multiprotease-deficient fission yeast by modification of its vacuolar protein sorting pathway. App/ Microbiol Biotechnol 2010, 85:667-677.

51. Yoon J, Aishan T, Maruyama J, Kitamoto K: Enhanced production and secretion of heterologous proteins by the filamentous fungus Aspergillus oryzae via disruption of vacuolar protein sorting receptor gene Aovps10. Appl Environ Microbiol 2010, 76:5718-5727.

52. Hermey G: The Vps10p-domain receptor family. Cell Mol Life Sci 2009, 66:2677-2689.

53. Quistgaard EM, Madsen P, Grøftehauge MK, Nissen P, Petersen CM, Thirup SS: Ligands bind to Sortilin in the tunnel of a ten-bladed $\beta$-propeller domain. Nat Struct Mol Biol 2009, 16:96-98.

54. Kelley LA, Sternberg MJ: Protein structure prediction on the Web: a case study using the Phyre server. Nat Protoc 2009, 4:363-371.

55. Kunz J, Schneider U, Deuter-Reinhard M, Movva NR, Hall MN: Target of rapamycin in yeast, TOR2, is an essential phosphatidylinositol kinase homolog required for G1 progression. Cell 1993, 73:585-596.
56. Sherman F: Getting started with yeast. Methods Enzymol 1991, 194:3-21.

57. Gould SJ, McCollum D, Spong AP, Heyman JA, Subramani S: Development of the yeast Pichia pastoris as a model organism for a genetic and molecular analysis of peroxisome assembly. Yeast 1992, 8:613-628.

58. Zacharias DA, Violin JD, Newton AC, Tsien RY: Partitioning of lipid-modified monomeric GFPs into membrane microdomains of live cells. Science 2002, 296:913-916.

59. Gietz RD, Sugino A: New yeast-Escherichia coli shuttle vectors constructed with in vitro mutagenized yeast genes lacking six-base pair restriction sites. Gene 1988, 74:527-534.

60. Gietz RD, Woods RA: Transformation of yeast by lithium acetate/singlestranded carrier DNA/polyethylene glycol method. Methods Enzymol 2002, 350:87-96.

61. Longtine MS, McKenzie A 3rd, Demarini DJ, Shah NG, Wach A, Brachat A, Philippsen P, Pringle JR: Additional modules for versatile and economical PCR-based gene deletion and modification in Saccharomyces cerevisiae. Yeast 1998, 14:953-961.

62. Goldstein AL, McCusker JH: Three new dominant drug resistance cassettes for gene disruption in Saccharomyces cerevisiae. Yeast 1999, 15:1541-1553.

63. Gaynor EC, Emr SD: COPI-independent anterograde transport: cargoselective ER to Golgi protein transport in yeast COPI mutants. J Cell Biol 1997, 136:789-802.

64. Bhave M, Papanikou E, lyer P, Pandya K, Jain BK, Ganguly A, Sharma C, Pawar K, Austin JI, Day KJ, Rossanese OW, Glick BS, Bhattacharyya D: Golgi enlargement in Arf-depleted yeast cells is due to altered dynamics of cisternal maturation. J Cell Sci 2014, 127:250-257.

doi:10.1186/s12934-014-0125-0

Cite this article as: Fitzgerald and Glick: Secretion of a foreign protein from budding yeasts is enhanced by cotranslational translocation and by suppression of vacuolar targeting. Microbial Cell Factories 2014 13:125.

\section{Submit your next manuscript to BioMed Central and take full advantage of:}

- Convenient online submission

- Thorough peer review

- No space constraints or color figure charges

- Immediate publication on acceptance

- Inclusion in PubMed, CAS, Scopus and Google Scholar

- Research which is freely available for redistribution

Submit your manuscript at www.biomedcentral.com/submit
C Biomed Central 